\title{
Cis-motifs upstream of the transcription and translation initiation sites are effectively revealed by their positional disequilibrium in eukaryote genomes using frequency distribution curves Kenneth W Berendzen ${ }^{1,2}$, Kurt Stüber ${ }^{1}$, Klaus Harter ${ }^{2}$ and Dierk Wanke*1,2
}

Address: ${ }^{1}$ Max-Planck Institute for Plant Breeding Research, Carl-von-Linné-Weg 10, D-50829 Köln, Germany and ²Universität Tübingen, ZMBP Pflanzenphysiologie, Auf der Morgenstelle 1, D-72076 Tübingen, Germany

Email: Kenneth W Berendzen - kenneth.berendzen@zmbp.uni-tuebingen.de; Kurt Stüber - stueber@mpiz-koeln.mpg.de; Klaus Harter - klaus.harter@zmbp.uni-tuebingen.de; Dierk Wanke* - dierk.wanke@zmbp.uni-tuebingen.de

* Corresponding author

Published: 30 November 2006

BMC Bioinformatics 2006, 7:522
Received: 23 May 2006

Accepted: 30 November 2006

article is available from: http://www.biomedcentral.com//47I-2/05/7/522

(C) 2006 Berendzen et al; licensee BioMed Central Ltd.

This is an Open Access article distributed under the terms of the Creative Commons Attribution License (http://creativecommons.org/licenses/by/2.0), which permits unrestricted use, distribution, and reproduction in any medium, provided the original work is properly cited.

\begin{abstract}
Background: The discovery of cis-regulatory motifs still remains a challenging task even though the number of sequenced genomes is constantly growing. Computational analyses using pattern search algorithms have been valuable in phylogenetic footprinting approaches as have expression profile experiments to predict co-occurring motifs. Surprisingly little is known about the nature of cis-regulatory element (CRE) distribution in promoters.

Results: In this paper we used the Motif Mapper open-source collection of visual basic scripts for the analysis of motifs in any aligned set of DNA sequences. We focused on promoter motif distribution curves to identify positional over-representation of DNA motifs. Using differentially aligned datasets from the model species Arabidopsis thaliana, Caenorhabditis elegans, Drosophila melanogaster and Saccharomyces cerevisiae, we convincingly demonstrated the importance of the position and orientation for motif discovery. Analysis with known CREs and all possible hexanucleotides showed that some functional elements gather close to the transcription and translation initiation sites and that elements other than the TATA-box motif are conserved between eukaryote promoters. While a high background frequency usually decreases the effectiveness of such an enumerative investigation, we improved our analysis by conducting motif distribution maps using large datasets.

Conclusion: This is the first study to reveal positional over-representation of CREs and promoter motifs in a cross-species approach. CREs and motifs shared between eukaryotic promoters support the observation that an eukaryotic promoter structure has been conserved throughout evolutionary time. Furthermore, with the information on positional enrichment of a motif or a known functional CRE, it is possible to get a more detailed insight into where an element appears to function. This in turn might accelerate the in depth examination of known and yet unknown cisregulatory sequences in the laboratory.
\end{abstract}




\section{Background}

The enormous amount of sequence data produced by various sequencing projects requires the development of suitable bioinformatic tools to help with functional characterization and annotation processes. One major problem in the analysis of non-coding DNA is the accurate assignment of functional attributes to defined stretches of sequence. For every gene, the sites for the initiation of transcription or translation are somehow encrypted in the DNA-sequence. Patterns of motifs define the positioning and the orientation of the basal transcription apparatus, which makes contact with the DNA at a certain position and is constituted by the RNA polymerase II complex and additional transcription factors. The DNAstretch essential for the recruitment of this multiproteincomplex is known to be the core promoter, a region that covers some hundred base-pairs flanking the transcription start site (TSS).

Accordingly, the sequences $5^{\prime}$ of the translation start codon ATG must encode for similar regulatory motifs that influence mRNA-abundance prior to, during and after transcription. As RNA elements embedded in the 5' untranslated regions (UTR) of the mRNA are much more complicated to study, only a few have been thoroughly characterized [1]. It is assumed that these motifs are the target sites for transcription factors or other regulatory proteins that directly or indirectly affect RNA-transcript or protein levels.

Although there are many cases known where genes remained conserved throughout organism kingdoms, only the TATA-box is mainly known to constitute a conserved motif in $30-40 \%$ of the core promoters of most species $[2,3]$. Besides the TATA-box, our knowledge and understanding of cis-regulatory elements (CRE) in different organisms is sparse and in most cases CRE databases if they exist at all - are specific to one organism. In contrast to the accessibility of sequence information via computational means, the possibilities for promoter analysis generally lag behind. Many computational programs exist that investigate promoter sequences either based on the frequency or on a positional bias [4-10]. In most cases proof of the functionality or importance of the identified motifs, in terms of a regulatory effect on transcript abundance, is lacking. The identification of sequence patterns involved in regulation is therefore an ongoing challenge for the in-depth understanding of gene expression.

Promising work has been done in the plant field with Arabidopsis thaliana, where the genome sequence is completely available, the amount of non-coding sequences is small, many regions close to the TSS are annotated and large CRE databases exist [11-14,53]. Additionally, in many genomes, particularly in eukaryotes, the majority of the sequences apart from the coding region do not provide useful biological information. Some improvement in the identification of regulatory motifs was gained by combining phylogenetic data with intensive expression profile analysis of co-regulated genes $[15,16,3]$. This approach is not universally applicable as either promoter sequence information on close phylogenetic relatives or the availability of expression data is lacking in many cases.

Large international sequencing programs have made the genome sequences for numerous prokaryotes and various eukaryote species available. Despite the fact that the number of correctly annotated genes is increasing [51], the amount of information is not yet sufficient to predict the TSS or the promoter for most of these genomes $[13,17,18]$. However, TSS prediction has been optimized up to $70 \%$ accuracy for human genes [19].

Here, we describe an enumerative approach for the identification of functional CREs via motif distribution maps in TSS or ATG rooted datasets. Moreover, we show that motif distribution maps constitute a universal means for the identification and characterization of motifs in promoter sequences of the model organisms Arabidopsis thaliana, Caenorhabditis elegans, Drosophila melanogaster and Saccharomyces cerevisiae. Our analysis identifies several motifs present in the promoters of all these eukaryote species, implying a universal promoter architecture that extends over various kingdoms and goes beyond our present image of promoter conservation.

The quality of our automated bulk extraction of promoters rooted with the TSS or the ATG was initially tested with known elements that are specific to each of the sets. Subsequently, novel promoter specific motifs have been discovered. To investigate previously known CREs, motif distribution maps were constructed, showing specific curve characteristics such as maxima and minima. Here, we focused on the analysis of conserved maxima only. Our results also support the quality of the genome sequences as such, and demonstrate that these are sufficient for bulk analysis of a large number of CREs.

\section{Results and discussion \\ Comparison of tetranucleotide sequences}

There are several recent publications on the identification of putative cis-regulatory elements based on a frequency bias or on the position of a certain DNA-motif $[4,17,7$ 10]. We decided to combine both methods by aligning sequences with respect to the annotated transcriptional start site (TSS) or the ATG and search for motifs that have alterations of both frequency and position. The motifs under investigation were plotted in respect to their position to form motif distribution maps, which were subsequently analyzed for positional over- or under- 
representation. A functional motif is defined by a frequency disequilibrium; a non random distribution at a precise position $[11,17,54]$. As a similar approach was undertaken earlier by Arkhipova with a preliminary, hand edited dataset from Drosophila melanogaster [20], we first attempted to reproduce her observations by using our script collection and the annotated genomic sequence available at the NCBI.

This analysis was performed on raw counts, but we have decided to display all graphs for reasons of comparability, as the percent of motifs relative to the total amount of sequences per dataset.

The distribution of the tetranucleotide sequences TATA and TCAG, which represent the palindromic TATA-box core and the cap-site initiator motif respectively, was analyzed by Arkhipova [20] in 252 Drosophila derived TSS rooted sequences spanning $-250 \mathrm{bp}$ to $+50 \mathrm{bp}$. On the basis of her work, Ohler et al. [21] determined the element distribution first in a frequency and subsequently in a position dependent manner in 1941 sequences using the same TSS region.

Here, we were able to extract 7954 Drosophila sequences surrounding the annotated TSS from $-250 \mathrm{bp}$ to $+50 \mathrm{bp}$. To get an insight into how well the TSS's are annotated in the databases, we used the 1941 sequences from Ohler et al. [21], whose accuracy was validated using BLAST searches [22].

Figure 1 shows motif distribution maps for TATA and TCAG derived from our dataset compared with the 1941 high quality, hand edited sequence set by Ohler et al. [21]. As expected, the TATA-motif exhibits its highest frequency at about $-28 \mathrm{bp}$, while the TCAG-motif is found to be relatively enriched exactly at the transcription initiation site. The observed background frequencies of the motifs in the

\section{-250 to +50}
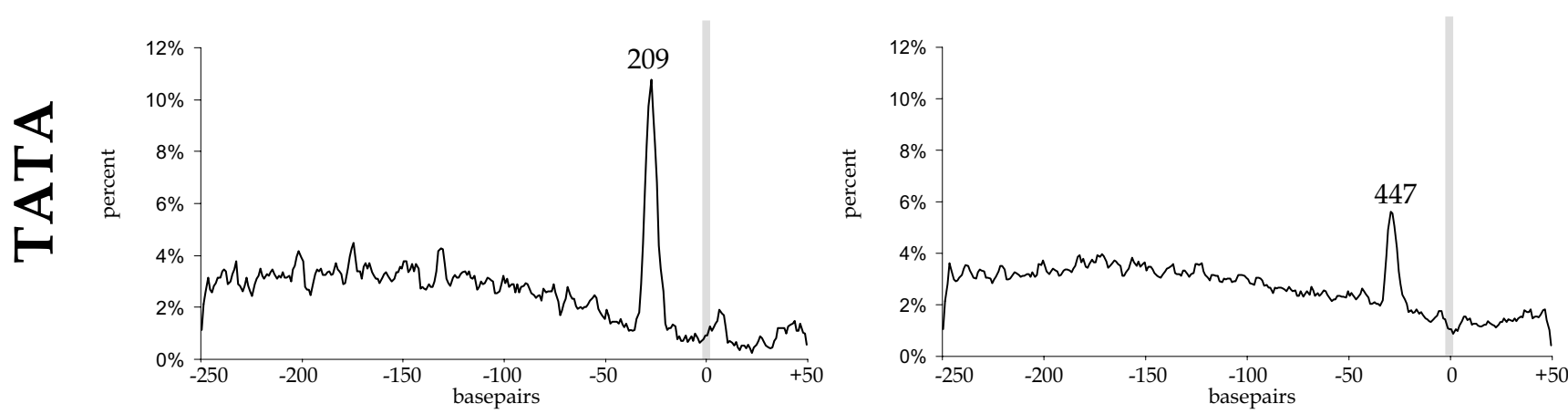

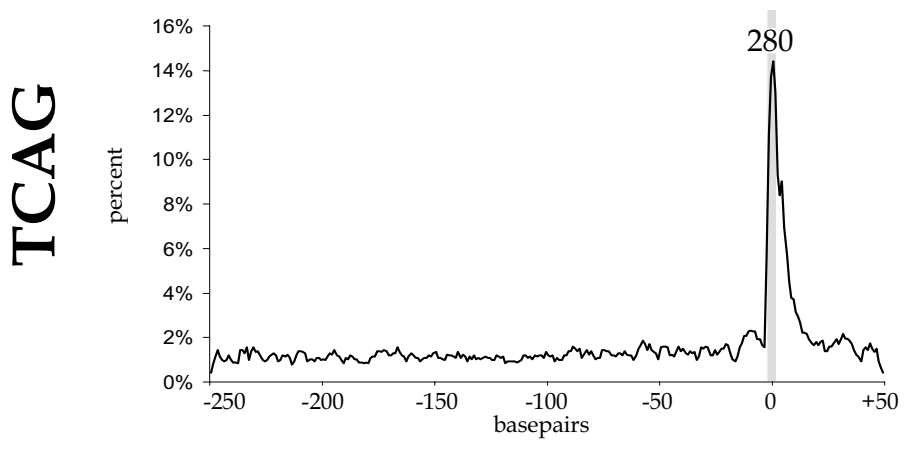

1941 sequences

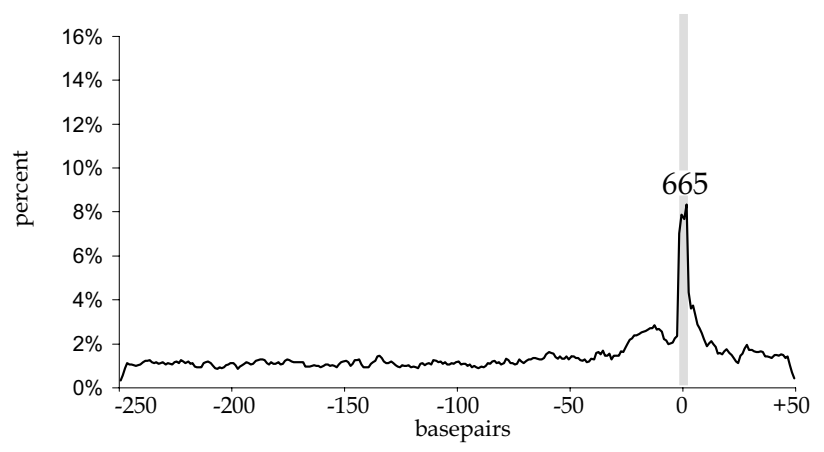

7954 sequences

\section{Figure I}

Comparison of the motif distribution curves for the TCAG and TATA motifs in Drosophila promoters. Previously examined motifs, TCAG and TATA [20], were mapped in a hand edited dataset constructed by Ohler et al. [2I] containing 1941 promoter sequences and an automatically assembled dataset of 7954 sequences derived from NCBI [59]. Relative number of motifs per site (in percent) was mapped to their respective position. Datasets spanning -250 bp to +50 bp were rooted with the position of the transcription start site (TSS) indicated as a grey bar. The absolute number of aligned sequences contributing to the maximum raw value of the peaks is given above the curves [see Additional File 7]. 
two datasets are of comparable percentages, whereas the relative peak height for the motifs of our GenBank dataset is about half of the Ohler et al. [21] dataset (Figure 1). The small portion of promoters containing a TATA-box and the conservation of its relative position coincide with previous works $[23,21,20]$.

\section{Positional disequilibria of functional regulatory motifs}

To get an impression of how much a motif is over-represented, we initially averaged over all counts per nucleotide position and calculated the standard deviation for the 250 bp to +50 datasets. We considered a peak to be significant if it deviated by four or more standard deviations from the background average. Additionally, to limit the computational work, we focused on the highest peak for each motif only.

For the dataset $-250 \mathrm{bp}$ to $+50 \mathrm{bp}$ rooted to the TSS we had to assume that coding sequences might influence this dataset, as 5'UTRs are sometimes shorter than 50 bp. To find out how specific the positional information on the four tetranucleotide sequences TATA, ATAA, TCAG and TCAT is with respect to the TSS, we analyzed their curve profiles in six different datasets (Table 1): two -250 bp to +50 bp datasets and four additional sets covering longer upstream regions $-1500 \mathrm{bp}$ to $+50 \mathrm{bp}$ and $-1500 \mathrm{bp}$ to -1 $\mathrm{bp}$, all of which were rooted to the TSS or the ATG.

As we wanted to exclude the TCAT-motif from overlapping with the ATG translation start codon in the ATG rooted datasets, we also included the CATG-motif in our analysis for comparison. If the TCAT motif were a part of the translation start site, we would expect to detect a peak right next to the ATG. The results in Table 1 demonstrate that the CATG-motif does not form a significant peak in any of the TSS rooted datasets, however TCAT does, which is indicative for the quality of the datasets' assembly with respect to the TSS. We are therefore confident that any short 5'UTR did not cause an informative peak detected in the TSS dataset.

Moreover, a significant overrepresentation of ATAA, TATA or TCAG was confirmed for the dataset used by Ohler et al. [21]. Under our strong confidence interval neither the palindomic TATA-motif nor the ATAA-sequence was considered to have a significant peak in both small -250 bp to +50 bp GenBank assembled datasets. This can be explained by the lower peak height in our datasets derived from GenBank (Table 1). Therefore, we computed a different background model using counts from -1494 bp to $494 \mathrm{bp}$ for each of the larger datasets (i.e. $\geq 1500 \mathrm{bp}$; Table $1)$. This reduced the background noise and we could prove that all four motifs investigated by Arkhipova [20] and Ohler et al. [21] are significantly enriched in all of the larger GenBank datasets. Remarkably, the TCAG/T ele- ment again generally marks the TSS while the TATA-box core is found about -30 bp upstream (Table 1).

The two related tetranucleotides, CATG which is predominantly found to constitute the translation start site and the TCAT-transcription initiation motif, carry the common trinucleotide CAT and can principally represent two different, non-overlapping molecular functions.

This hypothesis was resolved, when the translation start codon ATG was included in any of the sets, the CATGmotif was indeed the most prominent and overlapped with the position of the translation start site (Table 1). On the other hand, the CATG-motif did not have a peak in any TSS rooted dataset. Finally, if the translation start codon ATG was removed from the analysis in the large ATG rooted datasets of -1500 bp to $-1 \mathrm{bp}$, the CATG-motif was no longer significantly present. We concluded that we were able to differentiate clearly between similar motifs even with partially overlapping sequences. Hence, these similar motifs may encode different functions with respect to their position.

\section{Evolutionary conservation of the Inr motif}

An interesting question was, whether it might be possible to differentiate between the known functional TSS consensus (Inr) of Drosophila TCAKTY [24] and the more degenerate mammalian YYANWYY cis-regulatory element [25]. To address this question, we conducted a comparative motif analysis on our two D. melanogaster GenBank datasets spanning $-1500 \mathrm{bp}$ to $+50 \mathrm{bp}$ with these two motifs.

Mapping the TCAKTY motif in the 7954 Drosophila sequences rooted to the TSS, we found a distinct positional peak at the transcription initiation site (Figure 2). Interestingly, we could also identify a significant peak for the degenerate mammalian pattern YYANWYY in the Drosophila promoter sequences at about the same position. Although it was more frequent due to its degeneracy, the peak for the mammalian pattern YYANWYY still exceeds our strong confidence interval of 4 times the standard deviation over the background average. When a similar dataset of 10672 sequences aligned to the start codon ATG was used, both motifs lack significant curve profiles suggesting positional enrichment at the transcription start site only (Figure 2).

Our observations support the overall correctness of the annotated GenBank sequence data available, reaffirm that the specific Drosophila TSS is well characterized and illustrate a generally conserved nature of eukaryotic transcription information, since the mammalian TSS does yield a significant peak at the Drosophila TSS. 
Table I: Evaluation of tetranucleotide sequences for positional disequilibria in different Drosophila promoter datasets.

\begin{tabular}{|c|c|c|c|c|c|}
\hline Dataset & Element & Highest Peak (location) & SD above & BG-average & $S D$ \\
\hline \multirow[t]{5}{*}{ Ohler et al. [2I] } & ATAA & $220(-29)$ & 5.3 & 65.2 & 29.4 \\
\hline & CATG & no peak & - & 18.7 & 4.8 \\
\hline & TATA & $209(-30)$ & 5.8 & 49.7 & 27.6 \\
\hline & TCAG & $280(-2)$ & 7.6 & 31.3 & 32.7 \\
\hline & TCAT & $104(-1)$ & 6.6 & 28.9 & 11.3 \\
\hline \multirow[t]{5}{*}{ TSS $-250 . .+50$} & ATAA & no peak & - & 279.7 & 77.4 \\
\hline & CATG & no peak & - & 73.5 & 13.3 \\
\hline & TATA & no peak & - & 213.3 & 69.7 \\
\hline & TCAG & $665(-1)$ & 7.7 & 115.5 & 71.6 \\
\hline & TCAT & $268(-1)$ & 7.1 & 118.8 & 20.9 \\
\hline \multirow[t]{5}{*}{ ATG $-250 . .+50$} & ATAA & no peak & - & 322.4 & 102.4 \\
\hline & CATG & $2806(-1)$ & 8.5 & 124.8 & 315.9 \\
\hline & TATA & no peak & - & 224.6 & 79.8 \\
\hline & TCAG & no peak & - & 157.0 & 31.8 \\
\hline & TCAT & $628(-2)$ & 8.7 & 157.8 & 54.1 \\
\hline \multirow[t]{5}{*}{ TSS $-1500 . .+50$} & ATAA & $466(-3 I)$ & 17.6 & 196.2 & 15.3 \\
\hline & CATG & no peak & - & 105.7 & 10.6 \\
\hline & TATA & $447(-33)$ & 19.5 & 157.9 & 14.9 \\
\hline & TCAG & $665(-1)$ & 55.1 & 103.6 & 10.2 \\
\hline & TCAT & $268(-1)$ & 12.5 & 125.5 & 11.4 \\
\hline \multirow[t]{5}{*}{ ATG $-1500 . .+50$} & ATAA & $567(-3)$ & 12.0 & 285.3 & 23.5 \\
\hline & CATG & $2806(-1)$ & 204.1 & 136.2 & 13.1 \\
\hline & TATA & $357(-264)$ & 6.1 & 227.8 & 21.0 \\
\hline & TCAG & $243(-5)$ & 9.4 & 137.4 & 11.2 \\
\hline & TCAT & $628(-2)$ & 34.9 & 169.1 & 13.1 \\
\hline \multirow[t]{5}{*}{ TSS - I500..-I } & ATAA & $466(-26)$ & 17.6 & 196.1 & 15.3 \\
\hline & CATG & no peak & - & 105.7 & 10.6 \\
\hline & TATA & $447(-29)$ & 19.6 & 157.9 & 14.8 \\
\hline & TCAG & $226(-2 I)$ & 12.0 & 103.6 & 10.2 \\
\hline & TCAT & $174(-|04|)$ & 4.2 & 125.5 & 11.5 \\
\hline \multirow[t]{5}{*}{ ATG - $1500 . .-1$} & ATAA & $469(-196)$ & 7.8 & 285.2 & 23.5 \\
\hline & CATG & no peak & - & 136.2 & 13.1 \\
\hline & TATA & $357(-263)$ & 6.2 & 227.7 & 21.0 \\
\hline & TCAG & $243(-4)$ & 9.4 & 137.4 & 11.2 \\
\hline & TCAT & no peak & - & 169.1 & 13.1 \\
\hline
\end{tabular}

The hand edited dataset from Ohler et al. [2I] was compared with datasets based on annotated promoters that were extracted from genomic sequences derived from the NCBI [59]. The evaluation was conducted using motif distribution curves. Based on previous analysis [20], the significance cutoff frequency for a positional disequilibrium must differ by $\geq 4$ SD above the overall background average [see Additional File 8]. no peak: based on our evaluation, no significant peaks could be identified. 

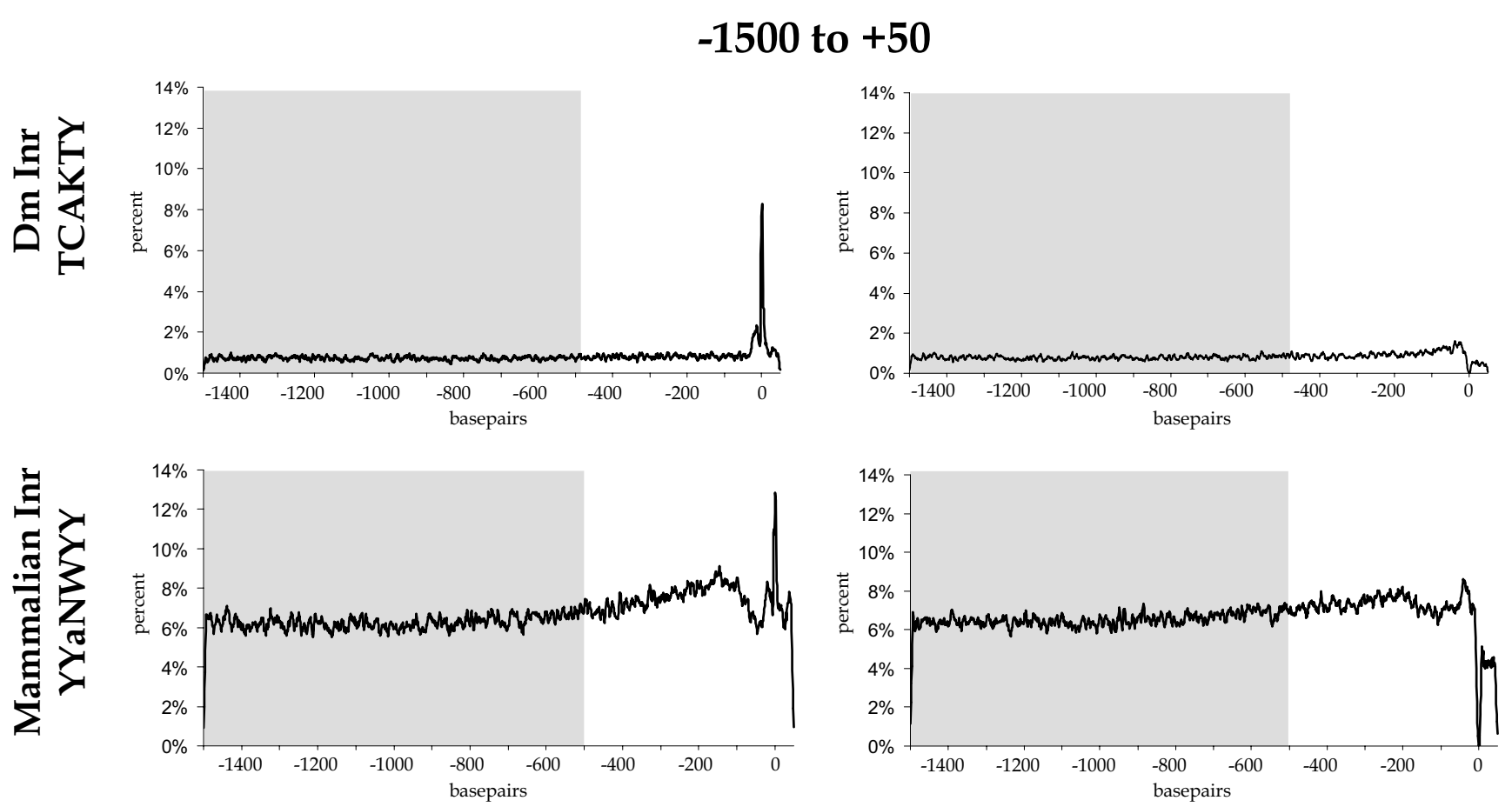

TSS

\section{ATG}

Figure 2

Distribution curves of the transcription initiation motifs from mammals and Drosophila in promoter datasets of Drosophila. The motif distribution curves of the transcription initiation motif (Inr) of Drosophila TCAKTY [24] and of mammals YYANWYY [25] were constructed on 7954 automatically assembled sequences derived from NCBI [59]. Relative number of motifs per site (in percent) was mapped to their respective position. The grey box indicates the region used to calculate the background average and its $S D$.

\section{Motif distribution curves on the TATA-box}

Although recent publications claimed that the annotated sequences at the primary DNA-databases were not good enough for unbiased bulk analysis of the TSS-upstream sequences [13], we concluded the opposite on the basis of the data presented so far. Hence, we broadened our analysis and acquired the sequences upstream of the TSS or the ATG for the model organisms Arabidopsis thaliana, Caenorhabditis elegans and Saccharomyces cerevisiae that are representatives of three organism kingdoms. As there were only very few genes in yeast that had an annotated TSS, we focused on the dataset aligned to the translation start codon ATG for this species only. As the most studied eukaryotic CRE is the TATA-box, we concentrated on the TATA-box hexanucleotides TATAAA and TATATA, as these motifs appear to form the functional TATA-box sequences at least for Drosophila and Arabidopsis [11,26,21,14].

To our knowledge no one has so far compared the positional occurrence of TATA-box sequences in different organisms. We expected no distinct disequilibria for both TATA-box motifs in the ATG rooted datasets for all four organisms; instead, we assumed that there would be a blurring effect caused by varying 5' UTR lengths. But the TATAAA motif formed clearly visible positional peaks in all of the datasets in the expected location (Figure 3), which was in agreement with other reports on TATA-box function $[13,26,24,23]$. The TATA-box motifs in S. cerevisiae have a wider breadth of enrichment and a double peak even though they are rooted to the ATG and not the TSS (Figure 3, see Additional File 1). This may be explained by the known fact that the TATA-box motif has a different positional dependence in S. cerevisiae (-120 bp to $-45 \mathrm{bp}$ instead of $-30 \mathrm{bp}$; [57]). Moreover, its relative distance to the ATG was comparable between the different organisms and, hence, the average size of the UTRs must be similar. Indeed, when we plotted the length distribution of the UTRs of Arabidopsis thaliana, Caenorhabditis elegans and Drosophila melanogaster, the UTRs of plant and the fly were similar in size, whereas the average C. elegans 

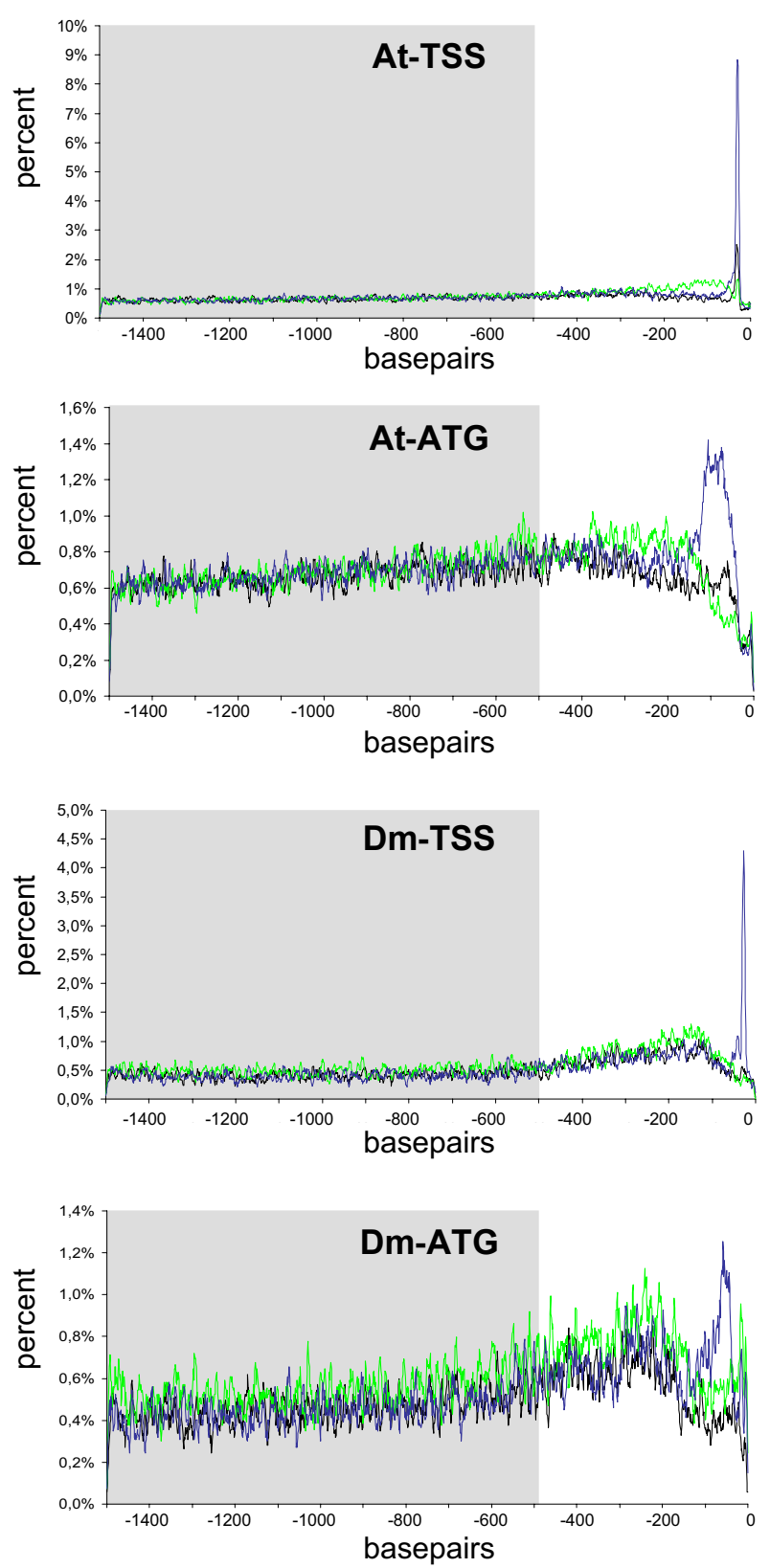
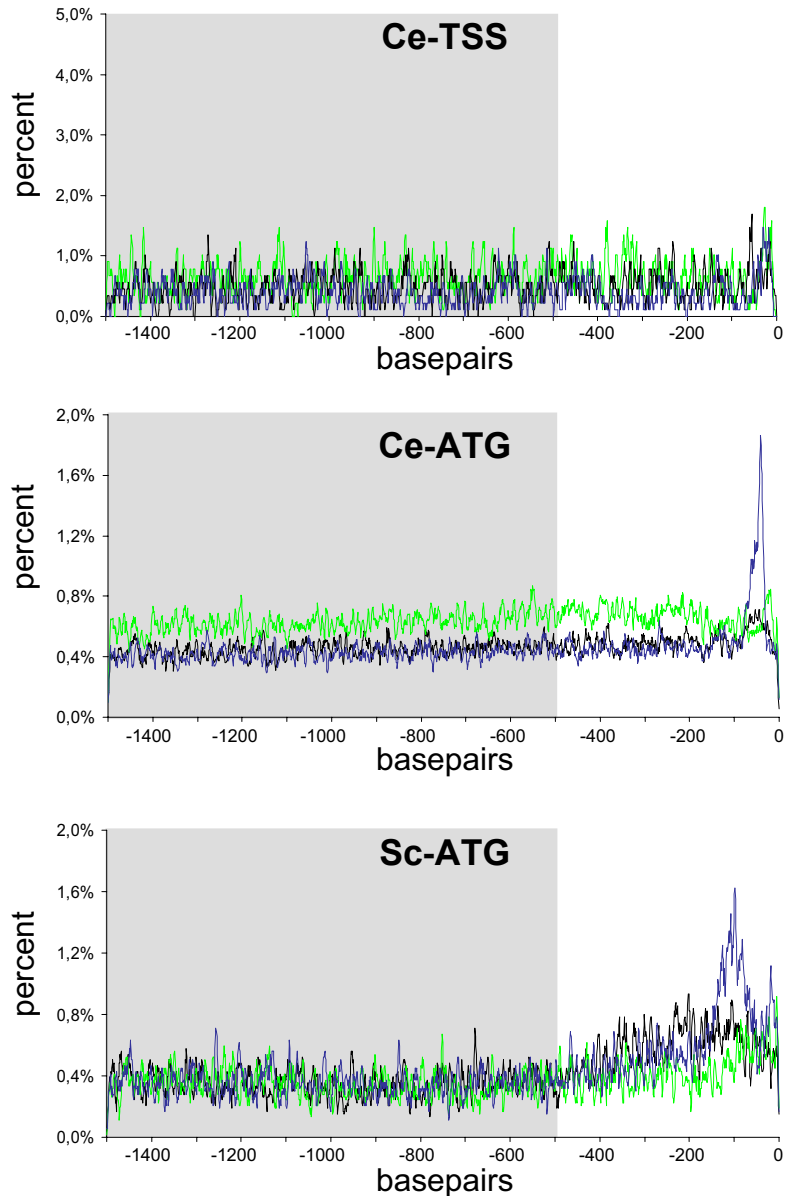

Figure 3

Distribution curves of the TATA-box motif TATAAA, its antisense TTTATA and the T/A-rich motif

ATTAAA. The motif distribution curves of the TATA-box hexanucleotide TATAAA, its antisense TTTATA and the T/A-rich motif ATTAAA were constructed on automatically assembled datasets of the Arabidopsis, Caenorhabditis, Drosophila and Saccharomyces genome sequences. Relative number of motifs per site (in percent) was mapped to their respective position. The grey box indicates the region used to calculate the background average and its $S D$. 
UTR was much shorter [see Additional File 2]. Saccharomyces is reported to have its average TSS at -15 bp to $-75 \mathrm{bp}$ before the ATG [23].

One important point is that the random frequency variation within the motif distribution maps decreases with the number of total sequences per dataset, which consequently affects the background based on the standard deviation. Therefore, the large Arabidopsis thaliana ATG rooted dataset with 26299 sequences was the most suitable to work with, while, in contrast, the C. elegans TSS rooted dataset with only 885 Sequences generated the largest amount of background noise.

To investigate the significance of the TATAAA motif, distribution curves were conducted for its antisense sequence TTTATA and a motif having the same A/T-composition, ATTAAA. As depicted in Figure 3, a subtle, non-significant peak could be seen for the TTTATA, even though it contains a TATA-signature and partially overlaps with the TATA-box. Furthermore, no significant maxima could be identified for the ATTAAA motif either. Thus, only the sense oriented TATAAA motif exhibits appositional disequilibrium.

The motif distribution maps conducted on the TSS aligned datasets revealed distinct peaks for TATA-box hexanucleotides for Arabidopsis as well as for D. melanogaster (Figure 3 and see Additional File 1). In the case of the TATAAA motif, peaks found for the two organism sets exceeded the average background distribution by 50 -fold or more. Only the TATAAA element formed an analyzable peak in the dataset from C. elegans, but only in the ATGrooted set (Figure 3). This might be explained either by the formation of multigene operons in Caenorhabditis, in which $15 \%$ of all genes underlying transcriptional regulation are organized as polycistronic pre-mRNA $[27,28]$ or by the existence of alternative TATA-like motifs with a degenerate nucleotide composition. Consequently, without this information the examined motifs can not be identified with significance.

To investigate the general occurrence of motifs carrying the same content of $\mathrm{T}$ and A mononucleotides, all 50 permutations of the TATAAA or TATATA hexamers were mapped in our reference datasets to examine if A/T-content alone could be detected by positional frequency disequilibrium (Table 2). Most of these T/A-rich hexanucleotides have regions in their respective distribution curves that differed by more than 4 times the standard deviation from their average background deviation depending on the analyzed dataset. Nearly all of the examined motifs were relatively enriched in the A. thaliana and D. melanogaster datasets rooted with the TSS as well as in the ATG-rooted set of D. melanogaster (Table 2).
In the single dataset of $S$. cerevisiae rooted to the ATG, 33 motifs still matched our stringent criteria, while in the corresponding $A$. thaliana set only 15 TA-hexanucleotides differed from their background frequency significantly. We found 29 different TA-permutations in both C. elegans datasets which were more frequent than their respective background model (Table 2). This varying enrichment of T/A-rich motifs can possibly be explained by different single nucleotide compositions close to the TSS or the ATG [see Additional File 3]. One other reason for the differences found might be the number of contributing sequences per dataset, as pointed out before.

However, many of these T/A-rich motifs exhibit significant enrichment, yet specific motifs have more significant tendencies than others, indicating a stronger positional bias. Among the most significant hexanucleotides in all organisms except for C. elegans, are the well known TATAbox elements TATAAA and TATATA (Table 2), which dominate the significance index. Additionally, the ATATAA-motif, which possibly overlaps with either of these TATA-box elements, was relatively enriched in the S. cerevisiae, D. melanogaster and C. elegans promoters. The TSS dataset for C. elegans did not reveal any of those motifs, but is rich in AATTTT or TAATTT (Table 2), which is consistent with its mononucleotide frequency profile (Additional File 4).

Hence, we can conclude that the motif analysis using frequency distribution maps from bulk sequence clearly identifies DNA-motifs containing various amounts of significant information. As we were able to validate previously characterized elements, we are convinced that this method of analysis gives important novel information on sequence data, as long as the amount of annotated genome sequence exceeds a certain minimal number of promoter sequences. We believe this threshold of a critical number of sequences will be specific for each organism.

\section{Hexanucleotide analysis with motif distribution curves in eukaryote promoters}

We next took an unbiased approach to validate the quality of the datasets and the value of our approach. Therefore, we expanded our analysis and conducted motif distribution maps for all 4096 hexanucleotides. First, the three TSS rooted datasets from Arabidopsis thaliana, Caenorhabditis elegans and Drosophila melanogaster were examined independently of the ATG rooted sets. Initially, the resulting 24576 frequency distribution maps were inspected for motifs that exhibit behavior signatures implying functional significance near the TSS or the ATG. In general, the majority of hexanucleotide motifs show non-variant background behavior in sequence upstream of $-600 \mathrm{bp}$ from the respective roots. This proves that the majority of sequence variation, and hence putatively encoded infor- 
Table 2: Analysis of all possible permutations of the TATA-box elements TATAAA and TATATA in the promoter sequences of the four model organisms.

\begin{tabular}{|c|c|c|c|c|c|c|c|}
\hline Element & Atl 500 tss & Atl500patg & ScI500atg & Dml500tss & Dml500atg & Cel500tss & Cel500atg \\
\hline TATAAA & 95.9 & 11.2 & 15.0 & 49.6 & 10.1 & 5.0 & 30.1 \\
\hline TATATA & 81.6 & 11.1 & 16.3 & 10.9 & 4.8 & 6.7 & 10.9 \\
\hline ATATAA & 67.7 & 6.8 & 15.1 & 18.0 & 5.2 & 6.7 & 15.5 \\
\hline ATAAAT & 52.0 & 4.9 & 8.3 & 12.2 & 6.0 & 4.7 & 5.9 \\
\hline ATATAT & 43.7 & 4.2 & 10.1 & 10.1 & 6.4 & - & 4.7 \\
\hline TAAATA & $4 I .4$ & 4.0 & 8.1 & 10.8 & 7.6 & 6.1 & 5.1 \\
\hline TTATAT & 27.0 & - & 7.2 & 9.7 & 4.7 & 5.7 & 8.1 \\
\hline TTTATA & 22.9 & - & 6.8 & 8.6 & 5.4 & 5.2 & 5.3 \\
\hline TTATAA & 22.5 & - & 6.2 & 8.7 & 6.3 & 4.8 & 4.2 \\
\hline TATTTA & 19.5 & 4.1 & 7.6 & 9.6 & 6.8 & 4.3 & 6.4 \\
\hline AATATA & 14.1 & - & 13.5 & 11.4 & 6.3 & - & 4.5 \\
\hline TATATT & 13.1 & - & 8.5 & 10.2 & 6.9 & 5.5 & 8.5 \\
\hline TTTAAA & 12.8 & - & 5.1 & 15.1 & 6.3 & 5.1 & 4.5 \\
\hline TATAAT & 12.3 & - & 5.4 & 7.9 & 5.3 & 4.8 & 5.9 \\
\hline TTAAAA & 12.1 & - & 7.9 & 12.7 & 7.5 & - & 4.2 \\
\hline ATTATA & 11.4 & - & 9.0 & 8.1 & 5.6 & - & - \\
\hline ATTTAA & 9.8 & 4.6 & 4.5 & 11.6 & 6.6 & 8.0 & 6.1 \\
\hline ATTTAT & 9.7 & - & 6.0 & 8.0 & 5.2 & 4.4 & 7.6 \\
\hline TTAAAT & 9.1 & - & 5.1 & 12.4 & 6.8 & 5.3 & 4.7 \\
\hline TAATAA & 9.0 & - & 7.3 & 10.9 & 7.1 & 6.7 & 4.5 \\
\hline TAAAAT & 8.8 & - & 4.5 & 13.0 & 6.6 & 6.5 & 4.6 \\
\hline ATTAAA & 8.5 & - & 6.7 & 9.6 & 6.5 & 4.4 & - \\
\hline TAAATT & 8.4 & - & - & 11.7 & 5.5 & 6.0 & 8.1 \\
\hline AAATAT & 8.2 & - & 8.6 & 10.5 & 5.5 & 6.5 & - \\
\hline TATTAA & 8.1 & - & 6.8 & 9.6 & 6.3 & 4.2 & 4.5 \\
\hline AATTAA & 7.7 & 4.2 & 9.7 & 10.0 & 5.8 & 4.6 & 4.5 \\
\hline ATAATA & 7.5 & - & 8.9 & 9.3 & 6.6 & 4.9 & - \\
\hline AATTTT & 6.3 & - & 9.6 & 10.7 & 5.5 & 16.5 & 7.7 \\
\hline TTAATA & 6.3 & - & 6.5 & 10.3 & 7.0 & 6.8 & 4.6 \\
\hline TTTTAA & 6.2 & - & 6.0 & 11.8 & 6.6 & 5.9 & 4.9 \\
\hline ATTAAT & 6.1 & 4.3 & 5.7 & 7.6 & 5.8 & 6.2 & 6.6 \\
\hline ATATTA & 5.9 & - & 5.7 & 9.8 & 6.2 & 4.1 & 4.0 \\
\hline AATAAT & 5.9 & - & 5.9 & 8.8 & 5.8 & 7.2 & 6.3 \\
\hline TTATTA & 5.9 & - & 5.4 & 8.7 & 5.5 & - & 6.3 \\
\hline TTAATT & 5.7 & 4.3 & 4.8 & 8.8 & 5.5 & 11.6 & 11.2 \\
\hline TAATTA & 5.7 & 4.1 & 6.5 & 7.4 & 5.9 & 5.2 & 5.3 \\
\hline AAATTA & 5.6 & - & 6.2 & 10.0 & 5.9 & - & - \\
\hline ATAATT & 5.3 & 4.4 & 5.9 & 9.3 & 5.5 & 5.7 & 6.7 \\
\hline AAAATT & 5.3 & - & 10.6 & 10.0 & 6.8 & 4.5 & - \\
\hline TTTAAT & 5.1 & 4.0 & 4.7 & 9.8 & 7.3 & 6.7 & 8.4 \\
\hline ATTATT & 5.0 & - & 6.3 & 9.5 & 6.2 & 5.2 & 9.3 \\
\hline AAATTT & 4.9 & - & 8.9 & 9.5 & 6.8 & 9.3 & 6.0 \\
\hline TATTAT & 4.8 & - & 7.3 & 8.2 & 5.6 & 4.7 & 5.6 \\
\hline AATATT & 4.8 & - & 5.7 & 9.6 & 6.1 & 9.5 & 8.0 \\
\hline TAATAT & 4.8 & - & 7.7 & 8.5 & 7.6 & 5.9 & 5.7 \\
\hline ATTTTA & 4.7 & - & 5.3 & 9.8 & 7.3 & 4.7 & 7.3 \\
\hline AATTAT & 4.6 & - & 4.6 & 8.0 & 5.5 & 5.2 & 5.4 \\
\hline ATATTT & 4.3 & - & 7.8 & 9.9 & 6.3 & 8.0 & 8.5 \\
\hline TAATTT & 4.3 & - & 5.1 & 11.1 & 6.0 & 14.3 & 11.5 \\
\hline AATTTA & 4.3 & 4.1 & - & 9.7 & 7.4 & 5.7 & 7.2 \\
\hline
\end{tabular}

Motif distribution curves were conducted for TATA-box like motifs and searched for frequency disequilibria that exceed a $\geq 4$ fold SD from the average. The fold SD differences over the background frequency at the position of the highest peak are given for each of the permutations [see Additional File 10]. Background average and SD were calculated as described in the material and methods. The table is sorted according to the fold differences found in the Arabidopsis promoters. 


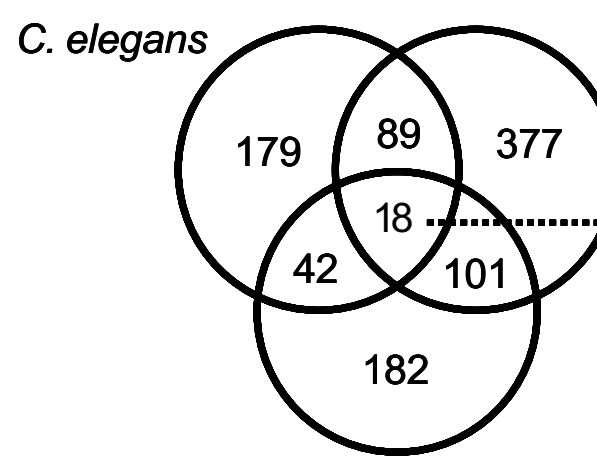

D. melanogaster
A. thaliana

\section{C. elegans}

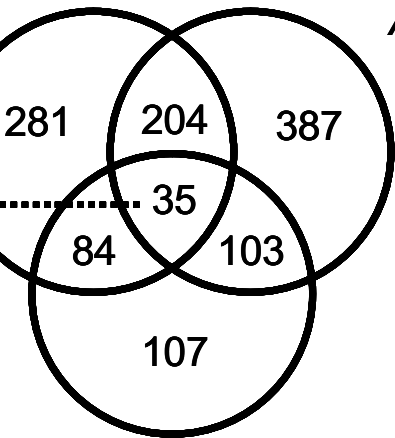

D. melanogaster

A. thaliana

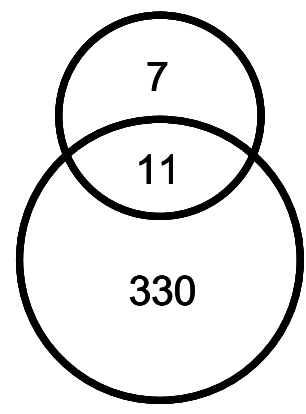

S. cerevisiae

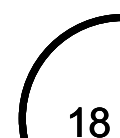

35
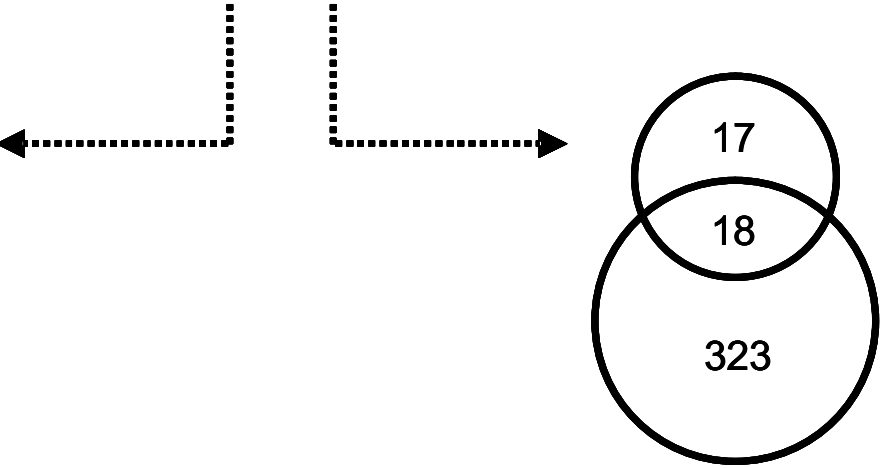

S. cerevisiae

\section{Translation Start Site}

\section{Figure 4}

Number of detected hexanucleotide motifs with a significant positional disequilibrium in different promoter datasets of the four model species. Diagram of hexanucleotide motifs enriched in the promoters of Arabidopsis, Caenorhabditis, Drosophila and Saccharomyces. Significant motifs exhibiting a relative positional disequilibrium that differed $\geq 6$ fold SD from the background average in promoter datasets spanning - I500 bp to - I bp of the transcription start site (TSS) or the translation start codon ATG [see Additional File 6]. Raw values of all significant hexanucleotides are given in Additional File II. Background averages and SD were calculated as described in the material and methods.

mation, is detectable near the TSS or the ATG. It is interesting to note that this observation had been made earlier $[17,29,30]$.

In contrast to the previous, more direct analysis of the functional TATA-box sequences where 4 times the standard deviation was significant, we needed to obtain overrepresented hexanucleotide motifs with more than 6 times standard deviation from the average background distribution, based on the varying background frequencies. This can be explained by a large number of rare or infrequent motifs that incapacitate the identification of disequilibria based on the evaluation of background standard deviations alone. There are 585 hexanucleotide motifs relatively enriched in the A. thaliana TSS dataset, 343 in the set of D. melanogaster and 328 in that one of $C$. elegans (Figure 4). In the ATG rooted datasets 729 motifs were overrepresented for $A$. thaliana, 604 and 329 for $C$. elegans and D. melanogaster, respectively.

It is noteworthy, that the number of motifs differing in the two sets was higher in the set rooted with the ATG. While the number of significantly enriched hexanucleotides identified for D. melanogaster did not change drastically, 
there are 1.8 fold and 1.2 fold more motifs for C. elegans and A. thaliana, in the ATG datasets compared to the TSS datasets, respectively (Figure 4). This might be explained by the presence of more putatively functional motifs in the 5'UTR regions of C. elegans and A. thaliana, implying an important and possibly underestimated regulatory function for the UTRs in these species.

Although about $8 \%$ to $10 \%$ of the possible 4096 hexanucleotides were motifs relatively enriched over the background frequency, only 18 were found to be commonly overrepresented in a promoter specific manner in the three TSS sets of all three species. In the corresponding sets rooted for the translation start codon (ATG), 35 common hexanucleotides were overrepresented in the promoter of A. thaliana, D. melanogaster and C. elegans.

To our surprise, none of the 18 motifs found in the TSS sets overlapped with any of those 35 hexanucleotides enriched in the datasets of the ATG (Figure 4), which in turn suggests that the datasets may contain different noncoding regulatory sequences. To get a preliminary insight into the hexanucleotide composition of $S$. cerevisiae promoters, we compared the ATG rooted dataset with the motifs shared in the promoters of the three previous organisms. Of the 18 motifs relatively enriched in the TSS datasets, 11 were also significantly enriched in the set from $S$. cerevisiae. Of the 35 motifs overrepresented in the ATG rooted sets, 18 were more frequent in the yeast dataset (Figure 4).
Table 3 gives a closer insight into the 18 motifs overrepresented in the sets rooted with the TSS of the three organisms compared with the ATG rooted set of $S$. cerevisiae. Besides T/A-rich motifs like the previously studied TATAlike motifs, the hexanucleotides CATTTT and TCACAC differed most with respect to their background frequencies and constitute putative functional CREs conserved in the promoters of all eukaryotes. It is noteworthy that both motifs, CATTTT and TCACAC, have recently been found to have a function in several animal promoters, including humans $[31,32]$.

Similarly, among the 35 motifs more frequent in the ATG rooted datasets, the TATAAA motif of the TATA-box is enriched in the promoters of all organisms (Table 4). Four motifs contain the TCAG-motif present in the transcription initiation sites studied in the beginning of this work. Besides A-rich sequences, six hexanucleotides exhibiting a TCAA-core sequence pattern and two a palindromic TGCA-core motif, both of unknown function in eukaryotes, were found in the list of significant hexamers (Table $4)$.

This analysis clearly identified several motifs that were not previously known to be conserved in promoters across organism kingdoms. Moreover, it proves the universal applicability of motif distribution curves for gathering useful positional information on promoter sequences.

Table 3: Motifs positioned at the transcription start site and shared between the four model organisms.

\begin{tabular}{|c|c|c|c|c|}
\hline Element & AtI500tss & Cel500tss & Dml500tss & Scl500atg \\
\hline TATATA & 81.6 & 6.7 & 10.9 & 16.3 \\
\hline ATATAA & 67.7 & 6.7 & 18.0 & 15.1 \\
\hline СТСТСТ & 43.3 & 6.2 & 8.1 & 4.2 \\
\hline TAAATA & 41.4 & 6.1 & 10.8 & 8.1 \\
\hline GTATAT & 25.8 & 8.0 & 11.2 & 7.8 \\
\hline ATTTAA & 9.8 & 8.0 & 11.6 & 4.5 \\
\hline CTCTCG & 9.4 & 8.1 & 6.2 & - \\
\hline GGCTAT & 9.2 & 6.1 & 10.8 & 4.3 \\
\hline TAATAA & 9.0 & 6.7 & 10.9 & 7.3 \\
\hline TAAAAT & 8.8 & 6.5 & 13.0 & 4.5 \\
\hline AAATAT & 8.2 & 6.5 & 10.5 & 8.6 \\
\hline TCACAC & 7.1 & 6.3 & 15.3 & 5.2 \\
\hline CATTTT & 6.5 & 14.8 & 6.4 & 7.6 \\
\hline AATTTT & 6.3 & 16.5 & 10.7 & 9.6 \\
\hline TTAATA & 6.3 & 6.8 & 10.3 & 6.5 \\
\hline TTATTT & 6.3 & 10.3 & 10.2 & 8.4 \\
\hline TTTATT & 6.1 & 8.9 & 10.4 & 7.6 \\
\hline ATTAAT & 6.1 & 6.2 & 7.6 & 5.7 \\
\hline
\end{tabular}

The standard deviation fold difference for the most significant peak is shown for each shared motif of the transcription start site datasets. Motif distribution curves were conducted and analyzed for all possible hexanucleotide motifs that have frequency disequilibria which exceeded $\geq 6$ fold $S D$ from the background average. Background average and SD were calculated as described in the material and methods. The table is sorted according to the fold differences found in the Arabidopsis promoters. 
Table 4: Motifs positioned at the translation start site and shared between the four model organisms.

\begin{tabular}{|c|c|c|c|c|}
\hline Element & AtI 500atg & Cel500atg & Dm/500atg & ScI500atg \\
\hline AGAAAA & 23.8 & 12.6 & 7.0 & 13.9 \\
\hline AAAGAA & 20.8 & 6.7 & 7.2 & 11.7 \\
\hline AACAAA & 16.2 & 12.7 & II.I & 12.8 \\
\hline AGCAAA & 15.6 & 13.0 & 15.6 & 13.0 \\
\hline ACAAAA & 15.4 & 13.3 & 7.0 & 13.2 \\
\hline ACAACA & 14.9 & 7.0 & 6.9 & 9.9 \\
\hline CAGAAA & 11.7 & 33.9 & 7.2 & 5.5 \\
\hline CAAACA & 11.3 & 6.6 & 6.9 & 9.5 \\
\hline TATAAA & 11.2 & 30.1 & 10.1 & 15.0 \\
\hline ATCGAA & 11.1 & 6.8 & 6.6 & 4.6 \\
\hline ССТАТА & 11.0 & 6.2 & 6.4 & 4.1 \\
\hline ATCAAA & 11.0 & 15.0 & 12.4 & 5.9 \\
\hline CTCAAA & 10.6 & 13.7 & 6.5 & - \\
\hline CAACAA & 10.4 & 12.2 & 10.5 & 10.9 \\
\hline TCAGAA & 10.3 & 47.7 & 7.0 & - \\
\hline CTATAA & 10.3 & 11.0 & 7.8 & 5.9 \\
\hline TCGATT & 9.5 & 6.2 & 6.2 & - \\
\hline CACAAA & 9.5 & 15.0 & $15 . \mid$ & 8.2 \\
\hline TGCAGA & 8.5 & 21.6 & 6.9 & - \\
\hline AACACA & 8.3 & 6.8 & 11.1 & 13.9 \\
\hline AATCAA & 8.3 & 11.9 & 13.6 & 6.6 \\
\hline TCAGCC & 8.2 & 21.6 & 7.3 & - \\
\hline TCAAAC & 8.0 & 9.8 & 6.9 & - \\
\hline CCCAAA & 7.7 & 9.3 & 8.0 & - \\
\hline TTTCAG & 7.6 & 121.5 & 7.3 & 4.7 \\
\hline TTGCAG & 7.5 & 34.0 & 11.0 & - \\
\hline CAATCA & 7.3 & 10.7 & 8.8 & 6.7 \\
\hline ATCAAC & 7.2 & 11.2 & 12.5 & 4.5 \\
\hline CCAAAA & 7.2 & 11.0 & 6.0 & 5.2 \\
\hline ACACAA & 7.2 & 8.9 & 10.9 & 12.2 \\
\hline CTTCAA & 7.0 & 11.6 & 7.5 & - \\
\hline TTCAGA & 6.7 & 90.1 & 7.0 & - \\
\hline TCAAAA & 6.7 & 14.1 & 6.4 & 5.3 \\
\hline ACCAAA & 6.6 & 7.8 & 12.3 & 5.1 \\
\hline ACAGAA & 6.2 & 9.9 & 10.2 & 5.7 \\
\hline
\end{tabular}

The standard deviation fold difference for the most significant peak is shown for each shared motif of the translation start site datasets. Motif distribution curves were conducted and analyzed for all possible hexanucleotide motifs that have frequency disequilibria which exceeded $\geq 6$ fold SD from the background average. Background average and SD were calculated as described in the material and methods. The table is sorted according to the fold differences found in the Arabidopsis promoters.

Motif distribution curves of known CREs from eukaryotes As the TATA-box constitutes a conserved functional motif, we were interested to expand this study and discover whether other known functional cis-regulatory elements also form positional peaks that could be investigated by using motif distribution curves. Therefore, we retrieved functional CREs in sense and anti-sense orientation from literature or public databases for transcription factor binding sites.

We were able to analyze 450 motifs from A. thaliana, 51 for S. cerevisiae and 87 for both, D. melanogaster and C. elegans, (Table 5; see Additional File 5) in their respective promoter datasets with the idea to explore whether or not known functional CREs other than known core-promoter elements can be identified using motif distribution analy- sis. Here as well, we expected a high variability in frequency amongst the different CREs and, hence, applied a significance cutoff for a positional disequilibrium of $\geq 6$ $S D$ difference from the background average for our automated analysis. Rare motifs, with a background average of $\leq 1$, were excluded. Taking into account that some of the boxes only function unidirectionally, for example the TATA-box, we scanned the promoter datasets for every CRE in both orientations. We found that at least 14,94\% of the known functional CREs do exhibit a significant positional disequilibrium matching our criteria (Table 5). The only exception was the TSS rooted set of C. elegans with only $8 \%$ of positional enrichment of CREs. This might again be explained by the formation of multigene operons in Caenorhabditis [27] or the limited amount of promoter sequence. In total, we observe that depending 
on the organism or the dataset investigated $8 \%$ to $50 \%$ of the putatively functional CREs were enriched at a defined position (Table 5). Besides T- or A-rich CREs, we found a significant increase in frequency for several motifs with a higher GC-content in both the TSS and the ATG rooted datasets. In about half the cases of a CRE showing a positional enrichment in any of the datasets, it was orientation dependent (Table 5). Other known functional core-promoter elements like the DPE, BRE or the MTE $[24,25,55]$ analyzed with datasets that extend over the transcription start sites could be detected only in Drosophila but not in the other species studied here (data not shown). Since the BRE and MTE are known to be enriched in TATA-less promoters [24], it may be worth performing a similar analysis with TATA-containing and TATA-less promoter datasets once more TSS information is available.

Although we have identified significantly enriched CREs with a positional disequilibrium on the basis of the peak height, valuable information is still retained in the frequency-distribution map of each motif, such as the breadth of enrichment.

Since it is impossible to depict frequency distribution curves for all CREs, we have randomly chosen two per organism that do not represent known core-promoter elements from our list of significant CREs for presentation (Figure 5). In the following paragraphs, we describe these examples in more detail.

\section{A. thaliana}

The 450 CREs for Arabidopsis were derived from the Plant cis-acting regulatory DNA elements (PLACE) database $[14,56]$. The GGCCCAWWW motif was found in genes induced after decapitating the inflorescences and might be a CRE for wound stress response and the initiation of axil- lary bud outgrowth in Arabidopsis [33]. The TGTCTC motif is known as the DR5-element and represents the binding consensus for the Auxin Response Factor 1 (ARF1) found in promoters of early auxin responsive genes [34]. This CRE is used as an important reporter element in a synthetic promoter to trace developmental processes in which the phytohormone auxin is involved [35-37]. Interestingly, the synthetic promoter contains a multimeric repeat of the TGTCTC consensus at a similar position where we identified a relative enrichment of this motif in our distribution curves.

\section{S. cerevisiae}

The 51 CREs for yeast were derived from the promoter database SCPD $[38,57]$. The CGTnnnnnnYGA motif represents the binding consensus for ABF1 [39]. The second motif, CGGGTRR, constitutes the binding motif for the REB1 protein [40]. Interestingly, both proteins are multifunctional, site-specific DNA-binding proteins that act in concert in many biological processes and are both involved in preventing gene expression at the silencing mating-type locus $[41,42]$. A positional enrichment of the binding consensi for both transcription factors about 100 to 200 bp upstream the ATG is congruent with their function as general regulatory factors at the transcription initiation site [43].

Since no specific CRE databases exist for C. elegans and $D$. melanogaster, we retrieved 87 CREs primarily from literature and textbook sources [see Additional File 5].

\section{C. elegans}

The BBTATWTWD consensus was identified in a phylogenetic footprint approach to form a regulatory module, which confers a muscle-specific gene expression [44]. The YTTCCTY motif is the binding consensus of ETS-transcription factors [45], which are associated with several physi-

Table 5: Number of known functional cis-regulatory elements (CRE) that have positional disequilibria.

\begin{tabular}{lcccc}
\hline Dataset & Number of Cis-elements & number over 6-SD & sing.ori/both.ori/palindrome & percentage over 6-SD \\
\hline Dm TSS & 87 & 13 & $2 / 10 / 1$ & $14,94 \%$ \\
Dm ATG & 87 & 14 & $8 / 5 / 1$ & $16,09 \%$ \\
& Dm shared & 12 & $6 / 5 / 1$ & $13,79 \%$ \\
At TSS & 450 & 77 & $33 / 35 / 9$ & $17,11 \%$ \\
At ATG & 450 & 71 & $38 / 27 / 6$ & $15,78 \%$ \\
& At shared & 33 & $11 / 17 / 5$ & $7,33 \%$ \\
Ce TSS & 87 & 7 & $7 / 0 / 0$ & $8,05 \%$ \\
Ce ATG & 87 & 44 & $26 / 16 / 2$ & $50,57 \%$ \\
& 7 & $7 / 0 / 0$ & $8,05 \%$ \\
Sc ATG & Ce shared & 16 & $7 / 8 / 1$ & $31,37 \%$ \\
\hline
\end{tabular}

Cis-elements [see Additional File 5] were retrieved and tested for positional disequilibrium as described in the text. CREs were analyzed for orientation bias (sing. ori = present in one orientation, both ori. = present in both orientations, or a palindrome). Percentage is the proportion of CREs relative to the respective total number of cis-elements investigated [see Additional File 12]. 


\section{A. thaliana}
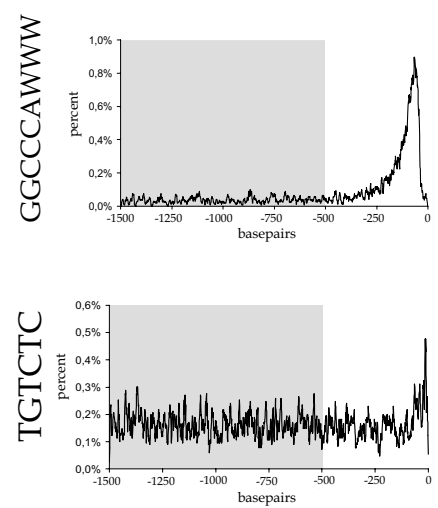

TSS

\section{D. melanogaster}
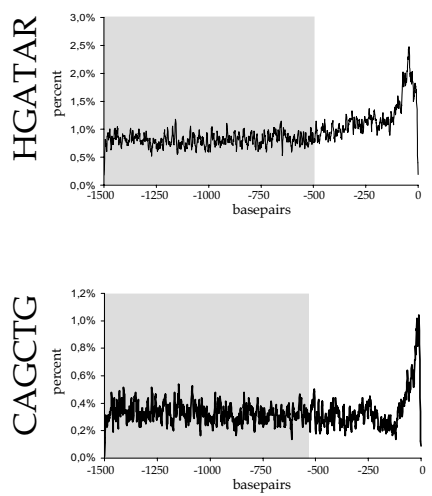

TSS
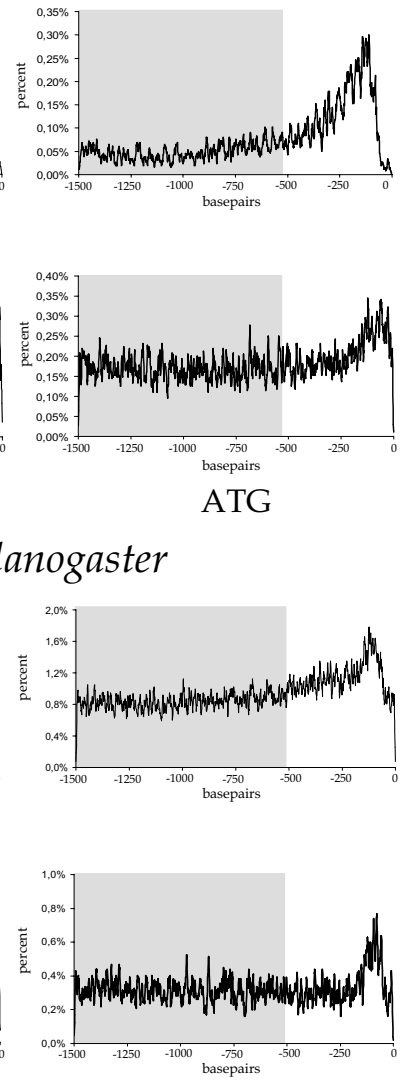

ATG

ATG
C. elegans
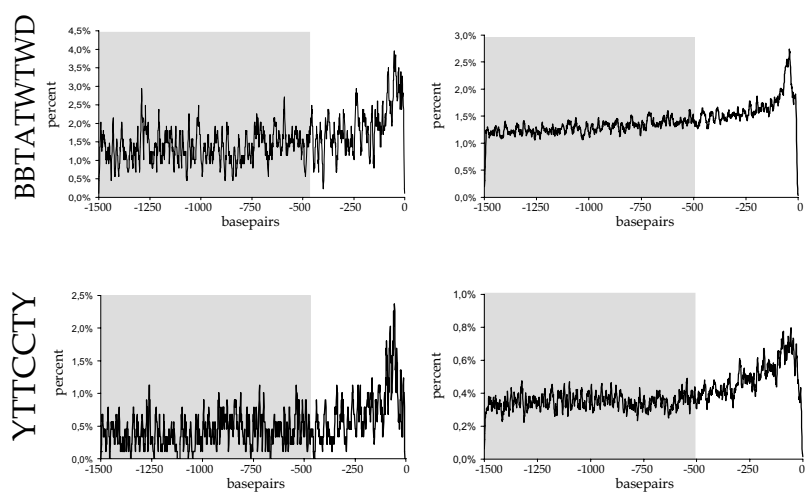

ATG

TSS

S. cerevisiae
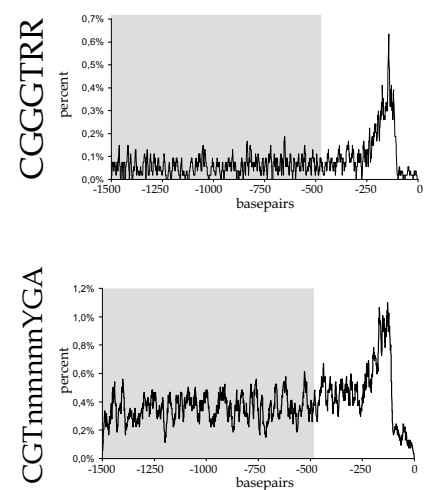

ATG

Figure 5

Examples of frequency distribution curves of known cis-regulatory elements (CREs). Two randomly chosen examples for CREs that possess positional disequilibria were shown for each of the model organisms. Known functional cis-regulatory elements of the three kingdoms (animals, fungi and plants) were taken from Table 5. Relative number of motifs per site (in percent) was mapped to their respective position. The grey box indicates the region used to calculate the background average and its SD. For raw values of the illustrated examples see Additional File I3.

ological and pathological processes. They contribute to the regulation of gene expression during the maturation of hematopoietic cell lineages and in tumor cell growth

\section{D. melanogaster}

The CAGCTG motif is known as the palindromic GC-box and represents a binding consensus conserved in 502 RNA polymerase II promoter regions [46]. The HGATAR motif was chosen as it was relatively enriched in Drosophila promoters, although it was described as a binding site for mesoderm specific MED1 GATA-factor in C. elegans [47]. This conforms to the idea that some cis-elements are conserved over large evolutionary distances and that our approach can identify such putatively functional motifs in eukaryote genome comparison approaches. For example, GAGA-like elements were first described in Drosophila and have recently been found to be functional elements in plants as well $[48,49]$.

\section{Conclusion}

It was proposed that the TATA-box was the only functional motif shared in the promoters between eukaryotes of different kingdoms [11] based solely on a frequency derived background model. Instead, our analysis has shown several highly conserved motifs besides TATA-box like patterns common in the promoter sequences flanking the TSS or the translation start codon ATG even amongst distantly related species.

Genome wide promoter analysis with frequency distribution maps expands our knowledge of the core promoter and the majority of known functional CREs. It has long been known that the mere presence of an element does not necessarily result in its functionality with respect to transcriptional regulation $[5,6]$. Hence, existing database information on CREs is valuable resource, i.e the PLACE collection of plant CREs [14] or the SCPD for yeast [38], 
but without positional information one could not reach any definite conclusion as to the significance of its putative functionality. With the information on positional enrichment of a motif or a known functional CRE it is possible to get a more detailed insight into where an element appears to function. This in turn might accelerate the in depth examination of regulatory sequences in the laboratory.

It has been shown that essential cis-regulatory modules exist in Drosophila melanogaster that control gene expression from a distance of several kilobases [50]. Nonetheless, we identify functionally important motifs to be located close to the TSS or the ATG. Whether such modules effect transcription via chromatin remodeling and nucleosome positioning, rather than a direct influence on the transcriptional machinery, remains to be examined.

On the basis of the average position of the TATA-box in the ATG rooted dataset, it also became evident that the 5'UTRs of different species can be of similar sizes, as was published before [see Additional File 2, [23]]. Moreover, some sequence pattern might be positionally enriched in the 5'UTR, implying an important and possibly underestimated regulatory function, but information on these kinds of elements is rare and needs more experimental investigation.

We concentrated on motifs which have maxima. But during this work, it became evident that motifs exist, which exhibit significant positional minima in the frequency distribution curves. Perhaps a detailed investigation of them could also contribute to our current understanding of gene regulation.

As our analysis was carried out on regular personal computers and the scripts were written in the Visual Basic script language, the analysis can be performed inexpensively and freely adopted or modified to suit specific needs. The universal applicability of our method and the simplicity of the background model used is a clear advantage of the presented approach. The use of motif distribution curves will greatly enhance the quality of the identification of novel promoter motifs, as many of the known functional CREs appear to be relatively enriched at defined sites near the core promoter region.

\section{Methods}

\section{Motif mapper}

The vast majority of the developed scripts mentioned in this work have been deposited in the Motif Mapper opensource script collection first released online in 2004 [58]. The Motif Mapper package is routinely maintained and up-dated at its web site, so that only the current version is available. A history file is available, documenting all changes and modifications made to the script code and package composition. Script version numbers mentioned refer to the individual script version number regardless of the package version which can be found in the beginning of each script file. Additionally, any older versions that have been replaced during package updates can be readily obtained upon request. Detailed descriptions accompany the package at its webpage.

\section{Extraction and alignment of sequences}

The GenBank DNA sequence flat-files were downloaded from the Entrez Plant Genomes Central at NCBI [59] for Arabidopsis thaliana [GenBank: NC 003070.5, GenBank: NC 003071.3, GenBank: NC 003074.4, GenBank: NC 003075.3, GenBank: NC 003076.4]; Drosophila melanogaster [GenBank: NC 004353.2.gb, GenBank: NC 004354.2.gb, GenBank: NT 033777.2.gb, GenBank: NT 033779.3.gb, GenBank: NT 037436.2.gb]; Saccharomyces cerevisiae [GenBank: NC_001133.5, GenBank: NC_001134.7, GenBank: NC 001135.3, GenBank: NC 001136.6, GenBank: NC 001137.2, GenBank: NC 001138.4, GenBank: NC 001139.5, GenBank:

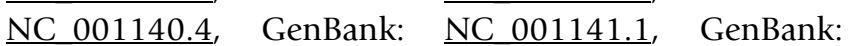
NC_001142.5, GenBank: NC_001143.5, GenBank: NC 001144.3, GenBank: NC 001145.2, GenBank: NC 001146.3, GenBank: NC 001147.4]; Caenorhabditis elegans [GenBank: NC 003279.2.gbk, GenBank: NC 003280.2.gbk, GenBank: NC 003281.3.gbk, GenBank: NC_003282.2.gbk, GenBank: NC_003283.3.gbk, GenBank: NC 003284.3.gbk]. Each chromosome sequence was extracted to an additional file using the SeqGBComplie v1.2 script.

Upstream regions rooted to the annotated transcription start site (TSS) or the translation start site (ATG) were extracted and compiled to FASTA formatted text with the aGenBankSQL script v3.3.4, using the default code modification suggested in the Motif Mapper website when extracting the TSS rooted datasets. This code modification only extracts promoters from genes that have an annotated or predicted 5'UTR. In cases where several TSS or ATG are annotated, the routines were adjusted in such a way that only the first annotation was recognized resulting in only one extracted sequence contributing to the promoter datasets. For each genome, the individual pseudo-molecule promoter datasets (i.e. each FASTA file per chromosome) were assembled into one file using the TxtFileFuse v1.2 script. Thereafter, frequency distribution curves were created for each motif by first combining the established promoter datasets with the StackerNtrimmer v.3.2.2 script to create the input files needed for the enumerating script REG_Pro_Point_Mapper v3.4.0. The number of extracted promoters is: At ATG 26299; At TSS 16997; Dm ATG 10671; Dm TSS 7953; Ce ATG 19179; Ce 
TSS 885; and Sc ATG 5361. A flowchart of the extraction and analysis is given as Additional File 4.

\section{Frequency analysis of motifs}

The REG_Pro_Point_Mapper algorithm identifies nonoverlapping motifs in each promoter from 5 ' to 3 ' orientation and records the position of the motif in its respective position in the promoter. Each sequence is searched independently and hits at the same position in two promoters are added together. A hexanucleotide hit is recorded as 111111 . Two overlapping hexamer motifs at identical positions in two different sequences result in 222222; hence, hexanucleotide motifs which overlap in their respective position by only one nucleotide in two different promoters are recorded as 11111211111 . Using this approach, we eliminated the need for a transformation to catch elements that have partial overlaps. Furthermore, simple smoothing algorithms tend to destroy the positional information in biological sequences (unpublished data). This is particularly evident when a motif, e.g. the translation start codon "ATG", is tightly positioned with respect to a biologically functional location within the DNA strand. Hence, models that average over a word size larger than the motif itself lose this positional information. Therefore, no smoothing operations have been applied to the data.

\section{Frequency distribution curves}

Frequency distribution curves were conducted on the textfile ".psum" output of REG_Pro_Point_Mapper v3.4.0 with regular spread sheet analysis programs. For each rooted dataset the raw number of motifs was plotted in respect to their exact position. As all sets used in this analysis were either rooted to the transcription start site (TSS) or the translation initiation codon ATG, all curves were drawn with the root pointing to the right.

\section{Detection of significant change in positional disequilibria}

Automatically evaluated motif distribution curves were searched for frequency disequilibria that exceed a defined fold standard deviation $(S D)$ from the average occurrence. We used the first $1000 \mathrm{bps}$ (-1494 bp to $-494 \mathrm{bp}$ ) from the $1500 \mathrm{bp}$ promoter sets to derive the background average, or the entire data set for all smaller datasets. The average background frequency constitutes the background model. We then calculated the standard deviation for each motif by using the following equation:

$$
S D=\sqrt{\frac{\sum\left(x_{i}-\bar{x}\right)^{2}}{(n-1)}}
$$

where $x_{i}$ is the number of counts at position $i, \bar{x}$ is the average from the background model and $n$ is the number of promoters in the dataset.
Assuming that motifs are equally distributed apart from any positional disequilibrium, we decided to use a simple significance cutoff frequency according to Arkhipova [20], defined as $\geq 4 S D$ from the average. Significant deviances from this average by 4 standard deviations were then used as a screening parameter to identify peaks and valleys. For evaluation on the curves for the 4096 hexanucleotides and known CREs, we introduced a higher cutoff frequency at $\geq 6 S D$ than the average of the background model. As certain motifs differ significantly in their frequencies near the transcription or translation start sites, but are not strictly position dependent (e.g. T/A rich motifs), the length of a significant stretch is recorded as a "gap". The script pSUMscan v.3.4.0 was written to automatically catalogue the peaks and valleys and gap data with the default set to 4 standard deviations (SD) above or below the background average; gaps were set to a default length of $25 \mathrm{bp}$. This entire automated analysis performed by the pSUMscan script can also be manually done in common spread sheet analysis programs.

\section{Cis-regulatory Elements (CREs)}

Known CREs were retrieved using the well maintained databases PLACE ([56]; 450 elements) for plant motifs and SCPD ([57]; 51 elements) for motifs from Saccharomyces. In order to gather CREs for Drosophila or Caenorhabditis, literature describing various eukaryotic cis-elements was collected and catalogued into a list of 87 elements. This list carries the original description of the cis-element, the IUPAC conversion used in this work and the original reference source describing the element. Apart from palindromes, we conducted motif distribution curves for sense and the antisense orientation of the motifs independently. The list of cis-elements used from PLACE, SCPD and other eukaryotes is found in Additional File 5.

\section{Motif permutations}

All possible permutations of the hexamers TATAAA and TATATA for each nucleotide were generated with the MAIN_AllCombinations v.3.4.1 script. Antisense motifs were generated by the MAIN_List_w_RevComps v.1.2 routine and all redundant motifs were removed with the script MAIN_ClearRedundancy v.1.2.

\section{Shared motifs}

Shared motifs were identified by conducting text file lists of overrepresented motifs for each dataset. The list content was compared using the SetGrouping script to identify shared motifs in both lists. Details can be found in Additional File 6.

\section{Abbreviations}

TSS, transcription start site; ATG, translation start site; $\mathrm{CRE}$, cis-regulatory element; $S D$, Standard Deviation; $A t$, 
Arabidopsis thaliana; Sc Saccharomyces cerevisiae; Dm, Drosophila melanogaster; Ce, Caenorhabditis elegans.

\section{Authors' contributions}

All authors have read and approved the manuscript.

DW and KS initiated the development of frequency-distribution analysis of cis-element distribution, KS and KB developed algorithms for analysis, KB programmed the Motif Mapper open source package, KH provided continuous support and research space for completion of this work.

\section{Additional material}

\section{Additional File 1}

Distribution curves of the TATA-box motifs TATAAA and TATATA. The motif distribution curves of the TATA-box hexanucleotides TATAAA and TATATA were constructed on automatically assembled datasets of the Arabidopsis, Caenorhabditis, Drosophila and Saccharomyces genome sequences. Relative number of motifs per site (in percent) was mapped to their respective position [see 1]. The grey box indicates the region used to calculate the background average and its SD.

Click here for file

[http://www.biomedcentral.com/content/supplementary/1471-

2105-7-522-S1.pdf]

\section{Additional File 2}

Size distribution of 5' UTRs. The 5'UTR lengths from each TSS dataset were plotted against their relative frequency.

Click here for file

[http://www.biomedcentral.com/content/supplementary/14712105-7-522-S2.pdf]

\section{Additional File 3}

Mononucleotide frequencies at the transition initiation sites TSS and ATG. The motif distribution curves of the four nucleotides were constructed on automatically assembled datasets of the Arabidopsis, Caenorhabditis, Drosophila and Saccharomyces genome sequences. Relative number of nucleotides per site (in percent) was mapped to their respective position.

Click here for file

[http://www.biomedcentral.com/content/supplementary/14712105-7-522-S3.pdf]

\section{Additional File 4}

Motif Mapper analysis flowchart. A flow chart of the sequence extraction and analysis procedure using Motif Mapper.

Click here for file

[http://www.biomedcentral.com/content/supplementary/14712105-7-522-S4.pdf]

\section{Additional File 5}

List of cis-regulatory elements and references. List of all cis-regulatory elements, their IUPAC version used in this work and their references. Click here for file

[http://www.biomedcentral.com/content/supplementary/14712105-7-522-S5.xls]

\section{Additional File 6}

Hexanucleotides positioned at the transcription or the translation start site and shared between the four model organisms. The standard deviation fold difference for the most significant peak is shown for each shared motif of the transcription or the translation start site datasets. Motif distribution curves were conducted and analyzed for all possible hexanucleotide motifs that have frequency disequilibria which exceeded $\geq 6$ fold SD from the background average.

Click here for file

[http://www.biomedcentral.com/content/supplementary/14712105-7-522-S6.xls]

\section{Additional File 7}

Raw data for Table 1. Contains the .psum-data for all of the motifs used in the D. melanogaster dataset comparisons between Ohler et al., (2002, [21]) and GenBank (2005).

Click here for file

[http://www.biomedcentral.com/content/supplementary/14712105-7-522-S7.xls]

\section{Additional File 8}

Peak analysis for Table 1. Peak analysis of 4-mer motifs used for D. melanogaster dataset comparisons between Ohler et al., $(2002$, [21]) and GenBank (2005), based on the raw information [see 1].

Click here for file

[http://www.biomedcentral.com/content/supplementary/1471-

2105-7-522-S8.xls]

\section{Additional File 9}

TATA-hexanucleotide comparison curves. Contains the .psum files and graphs for the elements TATATA and TATAAA for all genomes analyzed. Click here for file

[http://www.biomedcentral.com/content/supplementary/14712105-7-522-S9.xls]

\section{Additional File 10}

Peak analysis for the TATA-hexanucleotide comparison. Peak analysis files used for TATATA and TATAAA permutation analysis, including the summary table.

Click here for file

[http://www.biomedcentral.com/content/supplementary/1471

2105-7-522-S10.xls]

\section{Additional File 11}

All significantly enriched hexanucleotides. Peak analysis of all hexanucleotides that have SD fold differences of 4 or more for each dataset, including summary file.

Click here for file

[http://www.biomedcentral.com/content/supplementary/1471-

2105-7-522-S11.xls]

\section{Additional File 12}

Analysis of all known functional cis-regulatory elements. Peak analysis of all Cis-regulatory elements (CREs) that have SD fold differences of 4 or more for each dataset, including summary file.

Click here for file

[http://www.biomedcentral.com/content/supplementary/1471-

2105-7-522-S12.xls] 


\section{Additional File 13}

Raw data and graphs for Figure 5. Raw data on the two randomly chosen examples for CREs that possess positional disequilibria shown in Figure 5. Click here for file

[http://www.biomedcentral.com/content/supplementary/14712105-7-522-S13.xls]

\section{Acknowledgements}

We thank George Coupland for supporting Motif Mapper. We thank Felicity de Courcy for critical reading of the manuscript; and Joachim Kilian for technical help and discussions. This work was funded by the Deutsche Forschungs Gemeinschaft (SFB 446/AFGN HA2146/5-2).

\section{References}

I. Pesole G, Liuni S, D'Souza M: PatSearch: a pattern matcher software that finds functional elements in nucleotide and protein sequences and assesses their statistical significance. Bioinformatics 2000, 16:439-450.

2. Kutach AK, Kadonaga JT: The downstream promoter element DPE appears to be as widely used as the TATA box in Drosophila core promoters. Mol Cell Biol 2000, 20(13):4754-4764.

3. Katti MV, Sakharkar MK, Ranjekar PK, Gupta VS: TRES: comparative promoter sequence analysis. Bioinformatics 2000, 16(8):739-740.

4. Ben-Gal I, Shani A, Gohr A, Grau J, Arviv S, Shmilovici A, Posch S, Grosse I: Identification of transcription factor binding sites with variable-order Bayesian networks. Bioinformatics 2005, 2 I(I I):2657-2666.

5. Gershenzon NI, Stormo GD, loshikhes IP: Computational technique for improvement of the position-weight matrices for the DNA/protein binding sites. Nucleic Acids Res 2005, 33(7):2290-30I.

6. Zhou Q, Liu JS: Modeling within-motif dependence for transcription factor binding site predictions. Bioinformatics 2004, 20(6):909-916.

7. Matys V, Fricke E, Geffers R, Gossling E, Haubrock M, Hehl R, Hornischer K, Karas D, Kel AE, Kel-Margoulis OV, Kloos DU, Land S, Lewicki-Potapov B, Michael H, Munch R, Reuter I, Rotert S, Saxel H, Scheer $M$, Thiele S, Wingender E: TRANSFAC: transcriptional regulation, from patterns to profiles. Nucleic Acids Res 2003, $3 I(I): 374-378$.

8. Thijs G, Lescot M, Marchal K, Rombauts S, De Moor B, Rouzé P, Moreau $Y$ : A higher-order background model improves the detection of promoter regulatory elements by Gibbs sampling. Bioinformatics 200I, 17:III3-I I 22.

9. Van Helden J, Rios AF, Collado-Vides J: Discovering regulatory elements in non-coding sequences by analysis of spaced dyads. Nucleic Acid Res 2000, 28: $1808-1818$.

10. Hughes JD, Estep PW, Tavazoie S, Church GM: Computational identification of cis-regulatory elements associated with groups of functionally related genes in Saccharomyces cerevisiae. J Mol Biol 2000, 296: I205-I2/4.

11. Molina C, Grotewold E: Genome wide analysis of Arabidopsis core promoters. BMC Genomics 2005, 6:25.

12. Zhang W, Ruan J, Ho TH, You Y, Yu T, Quatrano RS: Cis-regulatory element based targeted gene finding: genome-wide identification of abscisic acid- and abiotic stress-responsive genes in Arabidopsis thaliana. Bioinformatics 2005, 2I(I4):3074-308I.

13. Shahmuradov IA, Solovyev VV, Gammerman AJ: Plant promoter prediction with confidence estimation. Nucleic Acids Res 2005, 33(3): 1069-1076.

14. Higo K, Ugawa $Y$, Iwamoto $M$, Korenaga T: Plant cis-acting regulatory DNA elements (PLACE) database. Nucleic Acids Res 1999, 27:297-300.

15. Chowdhary R, Ali RA, Albig W, Doenecke D, Bajic VB: Promoter modeling: the case study of mammalian histone promoters. Bioinformatics 2005, 2 I ( I I):2623-2628.
16. Wang T, Stormo GD: Combining phylogenetic data with coregulated genes to identify regulatory motifs. Bioinformatics 2003, 19:2369-2380.

17. Beer MA, Tavazoie S: Predicting gene expression from sequence. Cell 2004, I I7(2): 185-198.

18. Hudson ME, Quail PH: Identification of promoter motifs involved in the network of phytochrome A-regulated gene expression by combined analysis of genomic sequence and microarray data. Plant Physiol 2003, 133 (4):1605-1616.

19. Down TA, Hubbard TJ: Computational detection and location of transcription start sites in mammalian genomic. Genome Res 2002, I 2(3):458-46I.

20. Arkhipova I: Promoter elements in Drosophila melanogaster revealed by Sequence Analysis. Genetics 1995, 139:1359-1369.

21. Ohler U, Liao GC, Niemann H, Rubin GM: Computational analysis of core promoters in the Drosophila genome. Genome Biol 2002, 3( I 2):RESEARCH0087.

22. Altschul SF, Gish W, Miller W, Myers EW, Lipman DJ: Basic local alignment search tool. J Mol Biol 1990, 215(3):403-4I0.

23. Zang Z, Dietrich FS: Mapping of transcription start sites in Saccharomyces cerevisiae using 5' SAGE. Nucleic Acid Res 2005, 33(9):2838-285।.

24. Kadonaga JT: The DPE, a core promoter element for transcription by RNA polymerase II. Exp Mol Med 2002, 34(4):259-264.

25. Lim CY, Santoso B, Boulay T, Dong E, Ohler U, Kadonaga JT: The MTE, a new core promoter element for transcription by RNA polymerase II. Genes Dev 2004, I8(13): 1606-16I7.

26. Shahmuradov IA, Gammerman AJ, Hancock JM, Bramley PM, Solovyev VV: PlantProm: a database of plant promoter sequences. Nucleic Acids Res 2003, 3 I (I): I I4-I I 7.

27. Blumenthal T, Evans D, Link CD, Guffanti A, Lawson D, Thierry-Mieg J, Thierry-Mieg D, Chiu WL, Duke K, Kiraly M, Kim SK: A global analysis of Caenorhabditis elegans operons. Nature 2002, 4I 7(689I):85I-854.

28. Spieth J, Brooke G, Kuersten S, Lea K, Blumenthal T: Operons in C elegans: polycistronic mRNA precursors are processed by trans-splicing of SL2 to downstream coding regions. Cell 1993, 73(3):521-532.

29. Tompa $M$ : Identifying functional elements by comparative DNA sequence analysis. Genome Res 200 I, I I (7): I I43-I I 44.

30. Maleck K, Levine A, Eulgem T, Morgan A, Schmid J, Lawton KA, Dangl JL, Dietrich RA: The transcriptome of Arabidopsis thaliana during systemic acquired resistance. Nat Genet 2000, 26(4):403-4I0.

31. Spanagel R, Pendyala G, Abarca C, Zghoul T, Sanchis-Segura C, Magnone MC, Lascorz J, Depner M, Holzberg D, Soyka M, Schreiber S, Matsuda F, Lathrop M, Schumann G, Albrecht U: The clock gene Per2 influences the glutamatergic system and modulates alcohol consumption. Nat Med 2005, I I ( I):35-42.

32. Chen JR, Chatterjee B, Meyer R, Yu JC, Borke JL, Isales CM, Kirby ML, Lo CW, Bollag RJ: Tbx2 represses expression of Connexin 43 in osteoblastic-like cells. Calcif Tissue Int 2004, 74:56I-73.

33. Tatematsu K, Ward S, Leyser O, Kamiya Y, Nambara E: Identification of cis-elements that regulate gene expression during initiation of axillary bud outgrowth in Arabidopsis. Plant Physiol 2005, I38(2):757-766.

34. Ulmasov T, Murfett J, Hagen G, Guilfoyle TJ: Aux/IAA proteins repress expression of reporter genes containing natural and highly active synthetic auxin response elements. Plant Cell 1997, 9(II): 1963-197I.

35. Blilou I, Xu J, Wildwater M, Willemsen V, Paponov I, Friml J, Heidstra R, Aida M, Palme K, Scheres B: The PIN auxin efflux facilitator network controls growth and patterning in Arabidopsis roots. Nature 2005, 433:39-44.

36. Goda H, Sawa S, Asami T, Fujioka S, Shimada Y, Yoshida S: Comprehensive comparison of auxin-regulated and brassinosteroidregulated genes in Arabidopsis. Plant Physiol 2004, 134:1555-1573.

37. Casimiro I, Marchant A, Bhalerao RP, Beeckman T, Dhooge S, Swarup R, Graham N, Inze D, Sandberg G, Casero PJ, Bennett MJ: Auxin transport promotes Arabidopsis lateral root initiation. Plant Cell 200I, I3(4):843-852.

38. Zhu J, Zhang MQ: SCPD: a promoter database of the yeast Saccharomyces cerevisiae. Bioinformatics 1999, I 5(7-8):607-II. 
39. Lascaris RF, Groot E, Hoen PB, Mager WH, Planta RJ: Different roles for abflp and a T-rich promoter element in nucleosome organization of the yeast RPS28A gene. Nucleic Acids Res 2000, 28(6): $1390-1396$

40. Sjostrand JO, Kegel A, Astrom SU: Functional diversity of silencers in budding yeasts. Eukaryot Cell 2002, I(4):548-557.

4I. Yarragudi A, Miyake T, Li R, Morse RH: Comparison of ABFI and RAPI in chromatin opening and transactivator potentiation in the budding yeast Saccharomyces cerevisiae. Mol Cell Biol 2004, 24(20):9152-9164.

42. Graham IR, Chambers A: A Reb Ip-binding site is required for efficient activation of the yeast RAPI gene, but multiple binding sites for Rap Ip are not essential. Mol Microbiol I994, I 2(6):93 |-940.

43. Fourel G, Miyake T, Defossez PA, Li R, Gilson E: General regulatory factors (GRFs) as genome partitioners. J Biol Chem 2002, 277(44):4I736-4I743.

44. Wasserman WW, Fickett JW: Identification of regulatory regions which confer muscle-specific gene expression. J Mol Biol 1998, 278:|67-18|.

45. Mimeault M: Structure-function studies of ETS transcription factors. Crit Rev Oncog 2000, I I(3-4):227-253.

46. Bucher P: Weight matrix descriptions of four eukaryotic RNA polymerase II promoter elements derived from 502 unrelated promoter sequences. J Mol Biol 1990, 2I 2:563-578.

47. Broitman-Maduro G, Maduro MF, Rothman JH: The Noncanonical Binding Site of the MED-I GATA Factor Defines Differentially Regulated Target Genes in the C elegans Mesendoderm Developmental. Dev Cell 2005, 8(3):427-433.

48. Lehmann M: Anything else but GAGA: a nonhistone protein complex reshapes chromatin structure. Trends Genet 2004, 20(I):15-22.

49. Santi L, Wang Y, Stile MR, Berendzen KW, Wanke D, Roig C, Pozzi C, Muller K, Muller J, Rohde W, Salamini F: The GA octodinucleotide repeat binding factor BBR participates in the transcriptional regulation of the homeobox gene Bkn3. Plant J 2003, 34(6):8|3-826.

50. Berman BP, Nibu Y, Pfeiffer BD, Tomancak P, Celniker SE, Levine M, Rubin GM, Eisen MB: Exploiting transcription factor binding site clustering to identify cis-regulatory modules involved in pattern formation in the Drosophila genome. Proc Natl Acad Sci USA 2002, 99(2):757-762.

5I. FANTOM Consortium, RIKEN Genome Exploration Research Group and Genome Science Group (Genome Network Project Core Group): The transcriptional landscape of the mammalian genome. Science 2005, 309(5740): I559-63.

52. Hampsey M: Molecular genetics of the RNA polymerase II general transcriptional machinery. Microbiol Mol Biol Rev 1998, 62(2):465-503.

53. Molina C, Grotewold E: Genome wide analysis of Arabidopsis core promoters. BMC Genomics 2005, 6(I):25.

54. FitzGerald PC, Shlyakhtenko A, Mir AA, Vinson C: Clustering of DNA sequences in human promoters. Genome Res 2004, I 4(8): I562-74.

55. Gershenzon NI, loshikhes IP: Synergy of human Pol II core promoter elements revealed by statistical sequence analysis. Bioinformatics 2005, 2 I (8): 1295-300.

56. Plant cis-acting regulatory DNA elements (PLACE) database [http://www.dna.affrc.go.jp/PLACE/]

57. Saccharomyces cerevisiae Promoter Database [http:// rulai.cshl.edu/SCPD/]

58. Motif Mapper [http://www2.mpiz-koeln.mpg.de/coupland/coup land/mm3/html/]

59. National Center for Biotechnology Information [http:// www.ncbi.nlm.nih.gov/]
Publish with Bio Med Central and every scientist can read your work free of charge

"BioMed Central will be the most significant development for disseminating the results of biomedical research in our lifetime. "

Sir Paul Nurse, Cancer Research UK

Your research papers will be:

- available free of charge to the entire biomedical community

- peer reviewed and published immediately upon acceptance

- cited in PubMed and archived on PubMed Central

- yours - you keep the copyright
BioMedcentral 